\title{
ACESSO À EDUCAÇÃO POR FAIXAS ETÁRIAS SEGUNDO RENDA E RAÇA/COR
}

\author{
CIBELE YAHN DE ANDRADE \\ cyahn@terra.com.br
}

J. NORBERTO W. DACHS

ndachs@uol.com.br

Núcleo de Estudos de Políticas Públicas da Universidade Estadual de Campinas

$$
\text { RESUMO }
$$

São analisadas as contribuições da situação econômica e da raça/cor da pele no acesso à escola, até o ensino superior, considerando as principais transições escolares e grupos etários, usando dados da Pesquisa Nacional por Amostra de Domicilios - PNAD 2003. As variáveis renda e raça/cor afetam com intensidades diferentes as diversas faixas etárias e transições escolares. As restrições que levam ao reduzido percentual de jovens com acesso ao ensino superior dependem do número de vagas neste nível de ensino, mas parecem ser mais determinadas pelo pequeno contingente de jovens brasileiros que consegue completar o ensino médio, atingindo a qualificação formal necessária para o acesso ao ensino superior. Somente 40\% dos jovens de 18 a 24 anos possuem o ensino médio completo, sendo que 13\% tiveram acesso ao ensino superior. A situação econômica é um determinante mais importante do que a variável raça/cor, embora essa última variável apresente influência em todas as faixas de renda. Finalmente, estuda-se a distribuição dos alunos de ensino superior nesta faixa etária nos estabelecimentos públicos e privados.

ACESSO Ȧ EDUCAÇÃO - DISCRIMINAÇÃO NO ENSINO - CLASSE SOCIAL - RAÇA

\section{ABSTRACT}

ACCESS TO EDUCATION BY AGE GROUPS, ACCORDING TO INCOME AND RACE/ SKIN COLOUR IN BRAZIL. Data from the National Sample Household Survey - PNAD 2003 was employed to analyse the role of economic status and race/skin colour in access to school, including higher education. Income and race/skin colour variables have a strong impact on the various age groups and school transitions. Constraints that resulted in only a small percentage of young people having access to higher education are dependent upon the number of places the school has to offer at this level of education and even more by the reduced number of young Brazilians who manage to finish high school, achieving the required formal qualification to have access to higher education. Only 40\% of youths from 18 to 24 years of age complete high school and a mere $13 \%$ of which have access to college. The economic status plays a more important role than race/skin colour, although the latter has an influence throughout all income groups. Finally, the article analyzes the distribution of higher education students within this age group in public and private schools.

ACESS TO EDUCATION-EDUCATIONAL DISCRIMINATION-SOCIAL CLASS - RACE 
As desigualdades de oportunidade no mercado de trabalho dependem em grande medida das características de acesso ao sistema educacional (Camarano et al., 2004; Corseuil et al., 200 I; Osorio, 2004; Soares et al., 2005; Zuchi, 2004)'. Por sua vez, a situação econômica do grupo familiar, assim como a raça/cor dos indivíduos, reflete-se na sua evolução no sistema escolar. O número de estudos sobre a influência de cor/raça é mais numeroso (Beltrão, 2003; Beltrão, Teixeira, 2004; Soares et al.; 2005; Guimarães, 2003; Jaccoud, Beghin, 2002; Oliveira, 2005). São poucos, no entanto, os trabalhos que estudam esses dois determinantes de forma conjunta (Fernandes, 2004). Neste trabalho analisamos os efeitos da situação econômica do grupo familiar e da raça/cor no acesso ao ensino superior no Brasil, considerando também as principais transições escolares, por grupos etários, desde as séries iniciais do ensino fundamental, para identificar a seletividade que se observa no transcorrer do sistema educacional, usando como base os dados da PNAD/2003. Como medida da situação econômica se usa a renda familiar per capita e para raça/ cor se usa a classificação adotada pelo Instituto Brasileiro de Geografia e Estatística - IBGE.

Apesar do intenso crescimento da oferta do ensino superior, especialmente privado, ocorrido a partir da segunda metade da década passada, o percentual de jovens no Brasil que teve acesso a esse nível de ensino é ainda bastante reduzido. Segundo a PNAD/2003, apenas 13\% dos jovens de 18 a 24 anos freqüentam ou freqüentaram o ensino superior. $O$ debate sobre $O$ acesso a esse nível no Brasil, a partir do final da década de 90, passou a incluir, além das condições econômicas do grupo familiar, os efeitos relacionados à raça/cor dos estudantes. A situação econômica do grupo familiar nessa progressão e no acesso é considerada de forma indireta, ao serem propostos procedimentos

I Por exemplo, Soares et al. (2005) concluem que, apesar da relação entre a inserção no mercado de trabalho e o nível formal de instrução ser complexa e polêmica, algumas regularidades podem ser observadas. Em primeiro lugar, existe o fato, há muito tempo conhecido, de que, em média, quanto mais uma pessoa estuda, mais ela ganha. Pessoas com ensino médio ganham o dobro de pessoas com menos do que o ensino médio e metade do que ganham pessoas com alguma instrução superior. Além disso, o ensino médio é um passaporte para o mercado formal e garante pelo menos o salário mínimo, protegendo contra os empregos de salários irrisórios.

2 Para uma discussão sobre o conceito de pobreza, formas de medição e suas conseqüências, pode-se consultar Rocha (2003). 
Acesso à educação por faixas etárias...

de incentivo aos estudantes provenientes de escolas públicas de ensino médio (Jaccoud, Beghin, 2002; Moelecke, 2002; Maio, Santos, 2005; Maggie, Fry, 2004; Brandão, Marins, 2005; Pedrosa et al., 2006).

No debate sobre acesso ao ensino superior, um dos fatores pouco considerados é a existência de candidatos qualificados formalmente, isto é, com ensino médio completo. Como se verá a seguir, esta é uma restrição fundamental, especialmente para as camadas de renda mais baixa, independentemente de raça/cor, e que deve ser levada em conta nas estratégias de expansão do acesso de jovens brasileiros ao ensino superior.

No estudo das transições escolares são definidas para cada faixa etária as categorias de escolaridade adequada. Quanto ao acesso ao ensino superior são exploradas as suas diferenças, em relação aos sistemas públicos e privados, também segundo a renda familiar per capita e a raça/cor dos jovens.

As diferenças entre homens e mulheres em todos os níveis e para cada faixa de renda e também de acordo com a raça/cor são em geral pequenas, e deveriam ser tratadas em um estudo específico sobre o tema gênero (Beltrão, 2002).

\section{DADOS}

Os dados ${ }^{3}$ são da PNAD de 2003, inquérito de cobertura nacional e representativo para todas as unidades da federação. A renda per capita considerada é a do domicílio, sabendo-se que apenas aproximadamente $2 \%$ dos domicílios da amostra abrigam mais de um grupo familiar. Esta renda per capita é calculada dividindo a renda total do domicílio pelo número de moradores. O nível de escolaridade atingido e o acesso ao ensino superior são calculados usando as variáveis sobre freqüência atual ou anterior à escola, curso e série.

A informação sobre raça/cor pesquisada pelo IBGE é baseada na declaração do informante, tendo como referência a cor da pele, de acordo com as seguintes alternativas: "branco", "preto", "pardo" e "amarelo", além da categoria "indígena"

3. Os microdados de pesquisa são disponibilizados pela IBGE (http:www.ibge.gov.br/lojavirtual/ default.php).

4 Segundo Schwartzman (1999) "Existe muita insatisfação com estas categorias. Uma boa parte da população não se identifica e não gosta de alguns destes termos". Em relação aos percen- 


\section{METODOLOGIA}

Para a análise das transições escolares foram definidas as categorias de "escolaridade adequada", usando as informações de idade e série em que a pessoa está matriculada, ou o nível mais elevado que alcançou, conforme mostrado no quadro I. A forma usual é considerar separadamente a evasão, a repetência e o abandono em cada série e idade, mas aqui se optou pela definição dessas categorias de adequação por grupos etários, para permitir uma visão sintética da evolução dos indivíduos no sistema educacional.

Para a "escolaridade adequada" no ensino superior, considerou-se a faixa etária dos 18 a 24 anos, consistentemente com as estatísticas internacionais, o que permite a comparação das informações obtidas.

Para analisar o acesso ao ensino superior é importante não apenas calcular a taxa líquida em relação à população de 18 a 24 anos, mas também calcular a taxa de acesso ao ensino superior em relação aos jovens que são "formalmente qualificados para o ensino superior", ou seja, aqueles que conseguiram concluir o ensino médio, requisito formal necessário para o ingresso no ensino superior.

\section{QUADRO I}

DEFINIÇÃO DAS CATEGORIAS DE ESCOLARIDADE ADEQUADA

\begin{tabular}{|l|l|l|}
\hline Faixa etária & Ciclo escolar & Escolaridade adequada \\
\hline 7 a 10 anos & $I^{a}$ à $4^{a}$ série do EF & Freqüenta da $I^{\mathrm{a}}$ à $4^{\mathrm{a}}$ série do ensino fundamental \\
\hline II a I4 anos & $5^{\mathrm{a}}$ à $8^{\mathrm{a}}$ série do EM & Freqüenta da $5^{\mathrm{a}}$ à $8^{\mathrm{a}}$ série do ensino fundamental \\
\hline I5 a 17 anos & $E M$ & $\begin{array}{l}\text { Freqüenta o ensino médio; ou } \\
\text { Não Freqüenta, mas concluiu o ensino médio }\end{array}$ \\
\hline I8 a 24 anos & ES & $\begin{array}{l}\text { Freqüenta o ensino superior; ou } \\
\text { Não Freqüenta, mas teve acesso ao ensino superior } \\
\text { (tendo concluído ou não o curso) }\end{array}$ \\
\hline
\end{tabular}

tuais divulgados pelo IBGE ele afirma que "Os resultados que se encontram são também criticados... Estes números, segundo alguns críticos, ocultariam o verdadeiro tamanho da população negra no Brasil, que, se definida de forma análoga ao que ocorre nos Estados Unidos (segundo a origem étnica, no caso descendentes de africanos), chegaria a pelo menos 50\% da população; e também deixariam de medir o verdadeiro tamanho da população indígena". Para uma discussão em profundidade desse método de classificação e das diferenças em relação ao que é feito nos Estados Unidos da América pode-se ver Travassos e Williams (2004). 
Acesso à educação por faixas etárias...

As categorias usadas para a classificação da situação de escolaridade da população de 18 a 24 anos são mostradas no quadro 2 .

I. Os alunos que cursam o supletivo não foram considerados como tendo "escolaridade adequada", pois em 1996, a nova Lei de Diretrizes e Bases da Educação - LDB - aboliu a modalidade do ensino supletivo e instituiu o Educação de Jovens e Adultos - EJA -, ao mesmo tempo em que fixou a idade mínima de 15 anos, para prestar o exame conclusivo do ensino fundamental, e de 18 anos, para o ensino médio.

2. Não foram considerados na categoria "escolaridade adequada" aqueles que pararam de estudar aos 10 anos, mesmo tendo concluído a $4^{\text {a }}$ série na idade adequada. Da mesma forma, não foram considerados como tendo "escolaridade adequada" aqueles que pararam de estudar aos 14 anos, mesmo tendo concluído a 8 a série na "idade adequada".

Para a finalidade deste estudo, a população foi agregada em apenas dois grupos: "brancos" e "não-brancos". A razão principal para agregar as duas ca-

QUADRO 2

CLASSIFICAÇÃO DA SITUAÇÃO DE ESCOLARIDADE

DA POPULAÇÃO DE 18 A 24 ANOS

\begin{tabular}{|l|l|}
\hline Classificação da situação de escolaridade & Situação de escolaridade na PNAD \\
\hline Não concluiu o ensino fundamental & $\begin{array}{l}\text { Nunca freqüentou a escola } \\
\text { Evadiu-se sem concluir o ensino fundamental } \\
\text { Cursa o supletivo de ensino fundamental } \\
\text { Cursa o regular do ensino fundamental }\end{array}$ \\
\hline mas não concluiu o ensino médio & $\begin{array}{l}\text { Não freqüenta, mas concluiu ensino fundamental } \\
\text { Evadiu-se sem concluir o ensino médio } \\
\text { Cursa o supletivo do ensino médio }\end{array}$ \\
\hline Concluiu o ensino médio & Cursa o Regular do ensino médio \\
\hline (Freqüenta ou não) & Freqüenta curso pré-vestibular \\
\hline & $\begin{array}{l}\text { Não freqüenta atualmente, mas concluiu o ensino médio } \\
\text { Freqüenta o ensino superior }\end{array}$ \\
\hline Evadiu-se sem concluir o ensino superior \\
Não freqüenta atualmente, mas concluiu o ensino superior \\
Freqüenta ou freqüentou a pós-graduação
\end{tabular}


tegorias foi devida a motivos puramente técnicos, uma vez que o tamanho das amostras fica muito pequeno em certos grupos de idade e faixas de rendas. $\bigcirc$ grupo dos não-brancos é constituído por aqueles que se autodeclararam pardos (42\% do total da população), somados aos negros (6\%) e aos índios $(0,2 \%)$. O grupo dos brancos é composto pelos que se autodeclararam bran$\cos (52 \%$ do total da população) somados aos que se autodeclararam amare$\operatorname{los}(0,4 \%)$.

Para a classificação de rendimentos da população foi utilizada a renda familiar per capita, segundo frações do salário mínimo - SM - nas seguintes faixas: zero a menos de meio SM; meio a menos de um SM; um SM a menos de dois SMs; dois a menos de cinco SMs, e, mais de cinco SMs. $O$ valor do salário mínimo mensal em 2003 era de R\$240,00.

Para analisar o acesso ao ensino superior a estabelecimentos dos setores público e privado, além de tabelas e gráficos usuais, foi usada também a distribuição cumulativa do número de alunos que estudam nos dois setores de acordo com a renda per capita. Para isso se constroem curvas semelhantes à de Lorenz (que é usada para a renda), ordenando primeiramente os jovens de 18 a 24 anos de acordo com a renda per capita do domicílio e, então, acumulando no eixo horizontal a porcentagem de pessoas, e no eixo vertical, a porcentagem de matriculados. Para melhor comparar como são as duas distribuições, pode-se representar no mesmo gráfico a própria curva de Lorenz.

\section{RESULTADOS}

Para as faixas etárias consideradas, a situação de escolaridade é resumida na figura 1. Observa-se que para as crianças de sete a dez anos o acesso para as primeiras séries do ensino fundamental é praticamente independente da renda familiar e da raça/cor da população. Este resultado é basicamente uma conseqüência da quase universalização do ensino fundamental ocorrida no país nos últimos dez anos (Ferraro, Machado, 2002). Ainda assim, em torno de $10 \%$ dos não-brancos desta faixa etária, nas faixas de renda mais baixas, não apresentam "escolaridade adequada". O percentual de brancos em todas as faixas de renda é ligeiramente superior ao dos negros e pardos. 
FIGURA I

PERCENTUAL DA POPULAÇÃO COM "ESCOLARIDADE ADEQUADA" SEGUNDO A FAIXA ETÁRIA, RAÇA/COR E RENDA FAMILIAR PER CAPITA BRASIL, 2003

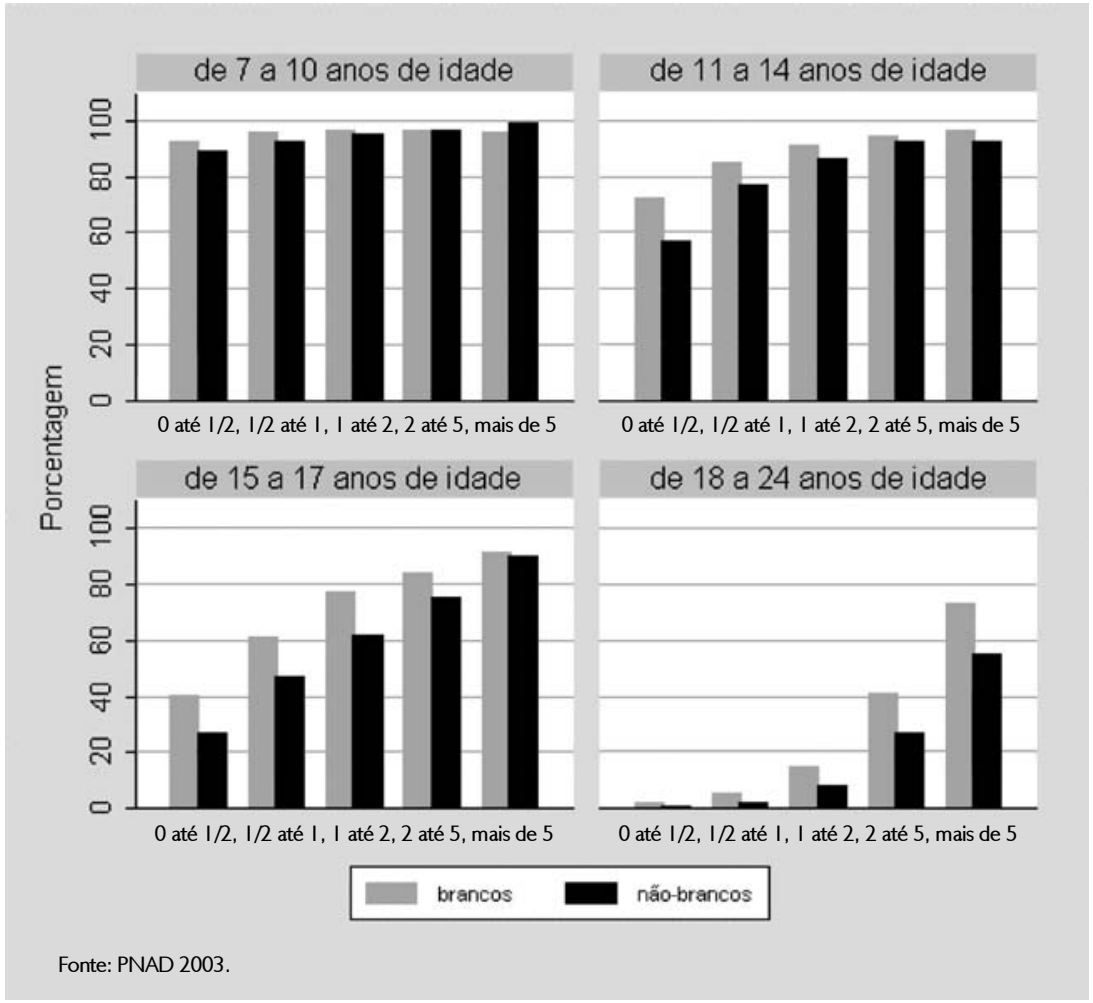

Na faixa etária de I I a 14 anos (nível de escolaridade adequado da $5^{\text {a à }}$ $8^{a}$ série), a situação observada se afasta da universalização; verifica-se uma variação mais acentuada em relação à renda e à raça/cor. Nas três faixas de renda inferiores (zero a meio, meio a um, e um a dois SMs) a diferença do acesso entre brancos e brancos é mais acentuada. Acima de dois SMs o percentual com "escolaridade adequada" se eleva para $90 \%$ dos jovens. A variação entre brancos e brancos ocorre em todas as faixas de renda desfavorecendo os nãobrancos, mas observa-se que as variações devidas ao fator raça/cor são bem menores do que aquelas relacionadas a faixa de renda. 
Na faixa etária de 15 a 17 anos (escolaridade adequada no ensino médio) as diferenças se acentuam ainda mais, com as variações do percentual de acesso, intensificando-se de acordo com a renda e também com a raça/cor. $\mathrm{Na}$ faixa salarial inferior, de zero a meio SM, são observados percentuais bastante baixos tanto para os brancos (30\%) como para os não-brancos (20\%). Na faixa de renda acima de cinco SMs, o percentual de acesso dos brancos e nãobrancos é próximo a 90\%, com ligeira deficiência para os últimos. Também nesta faixa etária as variações relacionadas ao fator raça/cor são bem menores do que aquelas relacionadas à faixa de renda, embora se observe sempre um desfavorecimento para os não-brancos.

Para os jovens de 18 a 24 anos, a variação dos percentuais de acesso ao ensino superior acentua-se, neste caso, tanto em relação à raça/cor como ao nível de renda, carregando claramente um efeito cumulativo das faixas etárias anteriores.

A figura 2 apresenta as porcentagens de pessoas no nível adequado de ensino, de acordo com faixas de renda e cor da pele. As curvas são apresentadas apenas para duas categorias de renda per capita, "alta" (mais de cinco SMs) e "baixa" (zero a meio SM). As outras três estão em posições intermediárias para estes extremos.

Em todos os grupos etários e para ambas as categorias de renda per capita nota-se que os não-brancos apresentam piores condições de escolaridade que os brancos, fato já amplamente documentado no Brasil (Barros et al, 200 I; Henriques, 200 I ). No entanto a renda familiar per capita constitui-se um determinante acima da variável raça/cor ${ }^{5}$, pois se observam diferenças bem menores entre brancos e não-brancos do que entre as categorias extremas de renda per capita. Hasenbalg e Silva (1999) demonstram em outras circunstâncias que a introdução de outras variáveis, tais como a distribuição regional da população, os anos de estudo do chefe do domićlio, chefia feminina e o ta-

5. Schwartzman (1999) associou a condição de raça/cor e anos de estudo dos indivíduos segundo o "rendimento médio mensal". Conclui que "é claramente a educação e não a cor, raça ou origem o grande fator de desigualdade na sociedade brasileira", porque a diferença de rendimentos encontrada entre os mais educados e os menos educados é de 9,9 vezes. Diferença muito maior do que a existente entre raças e cor (2,2 vezes), entre os que ganham mais (amarelos) e os que ganham menos (pardos) com o mesmo nível de escolaridade. 
FIGURA 2

PORCENTAGEM DE PESSOAS NO NÍVEL ADEQUADO DE ENSINO, DE ACORDO COM FAIXAS DE RENDA E COR DA PELE.

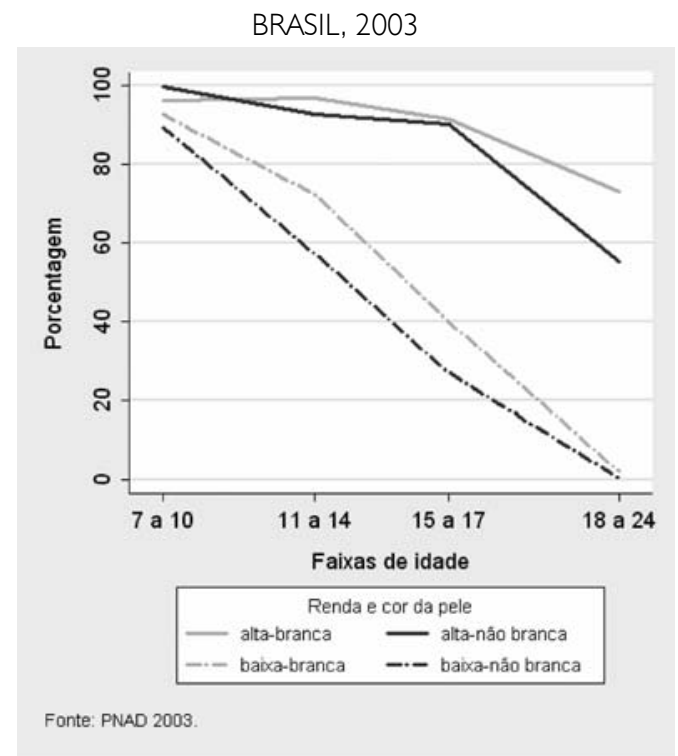

manho das famílias e especialmente a renda não eliminam o efeito da cor nas diferenças de acesso das crianças ao ensino formal, mas causam uma redução bastante considerável.

Olhando especificamente o grupo de 18 a 24 anos de idade nota-se que apenas 13\% desses jovens têm acesso ao ensino superior; torna-se relevante identificar a escolaridade daqueles que não atingem esse nível. Serão discutidas as associações do nível de escolaridade em relação à renda familiar, à cor/ raça dos indivíduos e os efeitos combinados desses dois determinantes.

A tabela I mostra a situação escolar da população nessa faixa etária, em função da renda familiar. É importante destacar que em toda a análise a categoria "concluiu o ensino médio" significa na realidade "concluiu no máximo o ensino médio", ou seja, não chegou a ter acesso ao ensino superior.

Sessenta por cento deles não possuem os requisitos escolares para o acesso ao ensino superior, pois 34\% não concluíram o ensino fundamental e $26 \%$ não concluíram o ensino médio. $\bigcirc$ contingente de jovens que estão formalmente qualificados e não tiveram acesso ao ensino superior é de apenas $27 \%$. 
TABELA I

NÍVEL DE ESCOLARIDADE DA POPULAÇÃO DE I 8 A 24 ANOS SEGUNDO A RENDA DOMICILIAR PER CAPITA

BRASIL, 2003

\begin{tabular}{|c|c|c|c|c|c|c|}
\hline \multirow[b]{2}{*}{ Níveis de escolaridade } & \multicolumn{5}{|c|}{ Renda familiar per capita em frações do salário mínimo } & \multirow[b]{2}{*}{ Total } \\
\hline & 0 a I/2 & $\begin{array}{l}\text { mais de } \\
1 / 2 \text { a I }\end{array}$ & $\begin{array}{c}\text { mais de } \\
\text { I a } 2\end{array}$ & $\begin{array}{c}\text { mais de } \\
2 \text { a } 5\end{array}$ & mais de 5 & \\
\hline Não concluiu o EF & $60 \%$ & $36 \%$ & $20 \%$ & $7 \%$ & $3 \%$ & $34 \%$ \\
\hline Concluiu o EF mas não EM & $26 \%$ & $32 \%$ & $28 \%$ & $17 \%$ & $9 \%$ & $26 \%$ \\
\hline Concluiu o EM mas não ES & $13 \%$ & $28 \%$ & $40 \%$ & $38 \%$ & $18 \%$ & $27 \%$ \\
\hline Acesso ao ES & $1 \%$ & $4 \%$ & $12 \%$ & $38 \%$ & $71 \%$ & $13 \%$ \\
\hline Total & 6.737 .444 & 5.941 .990 & 5.467 .768 & 3.272 .593 & 982.574 & 22.402 .369 \\
\hline \multicolumn{7}{|c|}{$\begin{array}{c}\text { TABELA } 2 \\
\text { NÍVEL DE ESCOLARIDADE DA POPULAÇÃO DE I } 8 \text { A } 24 \text { ANOS } \\
\text { SEGUNDO A RAÇA/COR } \\
\text { BRASIL, } 2003\end{array}$} \\
\hline \multirow{2}{*}{ Níveis de escolaridade } & \multicolumn{4}{|c|}{ Raça/Cor } & \multirow{2}{*}{\multicolumn{2}{|c|}{ Total }} \\
\hline & \multicolumn{4}{|c|}{ Não-brancos } & & \\
\hline Não concluiu o EF & $23 \%$ & & \multicolumn{2}{|l|}{$44 \%$} & \multicolumn{2}{|c|}{$34 \%$} \\
\hline Concluiu EF & $25 \%$ & & \multicolumn{2}{|l|}{$27 \%$} & \multicolumn{2}{|c|}{$26 \%$} \\
\hline Concluiu o EM & $31 \%$ & & \multicolumn{2}{|l|}{$23 \%$} & \multicolumn{2}{|c|}{$27 \%$} \\
\hline Acesso ao ES & $21 \%$ & & $5 \%$ & & \multicolumn{2}{|c|}{$13 \%$} \\
\hline Total & \multicolumn{2}{|c|}{11.665 .295} & \multicolumn{2}{|c|}{11.267 .262} & \multicolumn{2}{|c|}{22.932 .557} \\
\hline
\end{tabular}

A situação escolar desse grupo etário em função da renda familiar per capita é muito desigual. Na faixa inferior da renda, de zero a meio SM, o percentual dos jovens que sequer concluíram o ensino fundamental atinge mais da metade (60\%), enquanto na faixa de renda mais alta o acesso ao ensino superior ocorre para $70 \%$ dos jovens. $\bigcirc$ percentual de jovens que concluiu no máximo o ensino médio tende a ser menor, tanto na faixa de renda mais baixa (13\%), como na faixa de renda mais alta (18\%). Esses baixos percentuais parecem ter razões distintas: na faixa de renda mais baixa, são devidos ao grande percentual de jovens que não atingiu esse nível de escolaridade (60\% deles não concluíram sequer o ensino fundamental e os outros $26 \%$ não atingiram a última série do ensino médio); na faixa de renda mais alta, a maioria dos jovens 
Acesso à educação por faixas etárias...

(7|\%) já está cursando o ensino superior. Nas faixas intermediárias de renda, o percentual dos jovens que atingiram o ensino médio como o mais alto grau de escolaridade é maior, estando em torno de 30\% a $40 \%$.

Os dados da tabela 2 mostram que há acentuadas diferenças no nível de escolaridade atingido entre as populações dos jovens de 18 a 24 anos de idade, de raça/cor distintas. Essas diferenças são maiores nos dois extremos dos níveis de educação, ou seja, entre os que não concluíram o ensino fundamental e entre aqueles que tiveram acesso ao ensino superior. Entre os não-brancos, o percentual dos que não concluíram sequer o ensino fundamental é de aproximadamente $44 \%$, enquanto entre os brancos é de $23 \%$ e, para o acesso ao ensino superior os percentuais são de $21 \%$ entre brancos e apenas $5 \%$ para os não-brancos.

A tabela 3 e o gráfico da figura 3 apresentam as porcentagens de nível de escolaridade atingido pelos jovens de 18 a 24 anos de idade, até a conclusão do ensino médio, agora de acordo com níveis de renda e raça/cor da pele de maneira combinada.

$$
\begin{gathered}
\text { TABELA } 3 \\
\text { MAIOR NÍVEL DE ESCOLARIDADE ATÉ A CONCLUSÃO } \\
\text { DO ENSINO MÉDIO DA POPULAÇÃO DE I8 A } 24 \text { ANOS, } \\
\text { SEGUNDO A RENDA DOMICILIAR PER CAPITA E RAÇA / COR } \\
\text { BRASIL, } 2003
\end{gathered}
$$

Brancos

\begin{tabular}{lcccccc}
\hline \multirow{2}{*}{ Níveis de escolaridade } & \multicolumn{6}{c}{ Renda familiar per capita em frações do salário mínimo } \\
\cline { 2 - 6 } & $\mathbf{0}$ a I/2 & mais de & mais de & mais de & \multirow{2}{*}{ mais de $\mathbf{5}$} & \multirow{2}{*}{ Total } \\
& & I/2 a I & I a 2 & 2 a 5 & & \\
\hline Não concluiu o EF & $53,3 \%$ & $31,0 \%$ & $16,6 \%$ & $5,4 \%$ & $1,9 \%$ & $23,4 \%$ \\
Concluiu o EF mas não o EM & $28,0 \%$ & $33,6 \%$ & $27,5 \%$ & $16,1 \%$ & $7,6 \%$ & $25,1 \%$ \\
Concluiu o EM & $18,7 \%$ & $35,5 \%$ & $55,9 \%$ & $78,5 \%$ & $90,4 \%$ & $51,6 \%$ \\
\hline Total & 2.124 .199 & 2.693 .695 & 3.233 .697 & 2.472 .710 & 832.575 & 11.356 .876 \\
\hline
\end{tabular}

Não-brancos

\begin{tabular}{lcccccc}
\hline \multirow{2}{*}{ Níveis de escolaridade } & \multicolumn{6}{c}{ Renda familiar per capita em frações do salário mínimo } \\
\cline { 2 - 6 } & \multirow{2}{*}{ a l/2 } & mais de & mais de & mais de & \multirow{2}{*}{ mais de 5} & \multirow{2}{*}{ Total } \\
& & I/2 a I & I a 2 & 2 a 5 & & \\
\hline Não concluiu o EF & $62,8 \%$ & $40,7 \%$ & $23,9 \%$ & $12,7 \%$ & $6,9 \%$ & $44,1 \%$ \\
Concluiu o EF mas não o EM & $25,2 \%$ & $31,4 \%$ & $29,6 \%$ & $20,6 \%$ & $14,2 \%$ & $27,4 \%$ \\
Concluiu o EM & $12,0 \%$ & $27,9 \%$ & $46,5 \%$ & $66,6 \%$ & $78,9 \%$ & $28,5 \%$ \\
\hline Total & 4.613 .083 & 3.247 .803 & $2.234 .07 \mid$ & 799.883 & $\mid 49.999$ & $\mid 1.044 .839$ \\
\hline
\end{tabular}

Fonte: PNAD, 2003. 
FIGURA 3

MAIOR NÍVEL DE ESCOLARIDADE ATÉ A CONCLUSÃO

DO ENSINO MÉDIO DA POPULAÇÃO DE I 8 A 24 ANOS SEGUNDO A RENDA DOMICILIAR PER CAPITA E RAÇA /COR

BRASIL, 2003

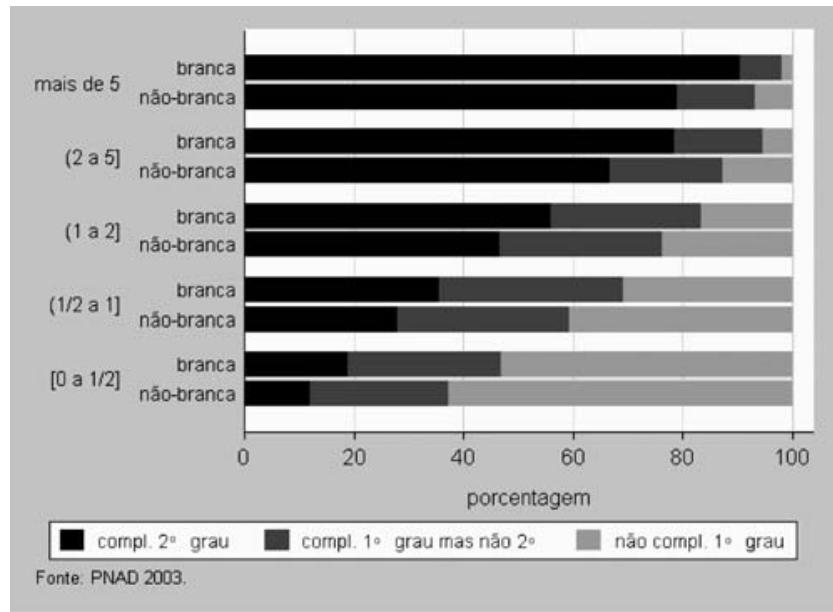

Quanto à conclusão do ensino médio as diferenças entre os dois grupos é semelhante em termos absolutos em todas as faixas de renda, o que significa maior diferença relativa nas faixas de renda inferiores, sendo, por exemplo, de 18\% e 12\% para renda per capita até meio salário mínimo e de $90 \%$ e 79\% para rendas superiores a cinco salários mínimos. Esse atraso do grupo dos não-brancos se deve principalmente às elevadas taxas de não conclusão do ensino fundamental que são de $53 \%$ e $63 \%$ entre brancos e não-brancos na faixa de renda mais baixa e de $2 \%$ e $7 \%$ na faixa mais alta. As diferenças elevadas observadas entre brancos e não-brancos na população geral (para todas as faixas de renda - Tab. 2) se devem à maior concentração da população não-branca nas faixas de rendas mais baixas. São 7,8 milhões de jovens com rendas per capita inferiores a um salário mínimo enquanto são 4,8 milhões de brancos na mesma faixa de renda, justamente aquela em que os níveis de escolaridade são os mais baixos. No extremo superior, na faixa de mais de cinco salários mínimos per capita, são apenas 150 mil não-brancos, comparados com 833 mil representando, respectivamente, cerca de 1,4\% e 7,3 \% dos nãobrancos e brancos desse grupo de idade. 
Acesso à educação por faixas etárias...

A tabela 4 e o gráfico da figura 4 mostram as porcentagens de jovens que tiveram acesso ao ensino superior em relação ao contingente daqueles formalmente qualificados, ou seja, que concluíram o ensino médio.

Novamente, as diferenças entre as porcentagens de brancos e não-brancos são semelhantes em todas as faixas de renda, oscilando entre 7,7\% (12,4 $4,7)$ nas rendas mais baixas, a 10,6\% (8I - 70,4) nas rendas mais altas. No entanto, nesse caso, mais ainda do que anteriormente, as diferenças relativas são muito maiores na primeira faixa de renda, caindo gradativamente até a última. Para a população de 18 a 24 anos de idade com renda per capita até um salário mínimo a porcentagem de brancos que tem acesso ao ensino superior é mais do que duas vezes e meia maior do que para não-brancos, enquanto na faixa de mais de cinco salários mínimos de renda per capita é apenas I, I 5 vezes maior.

Na tabela 5 se apresenta a distribuição das matrículas no ensino superior público e privado segundo a condição de renda da população. Do total de 2,4 milhões de jovens de 18 a 24 anos que cursavam o ensino superior em $2003,72 \%$ encontram-se no ensino superior privado e $28 \%$ no ensino superior público.

Do total de matriculados na rede privada, apenas $2 \%$ se situam na faixa de renda familiar per capita de menos de meio salário mínimo per capita, enquanto dos que estão na rede pública nesta faixa de renda, situam-se $5 \%$ do total de matriculados. As distribuições por nível de renda na rede pública e privada são bastante semelhantes na parte central dos níveis de renda. Até rendas de dois salários mínimos per capita se encontram 30\% dos matricula-

TABELA 4

PORCENTAGEM DOS JOVENS DE I 8 A 24 ANOS COM ACESSO AO ENSINO SUPERIOR ENTRE OS QUE CONCLUIRAM O ENSINO MÉDIO, SEGUNDO A RENDA DOMICILIAR PER CAPITA E RAÇA / COR, BRASIL, 2003

\begin{tabular}{|c|c|c|c|c|c|c|}
\hline \multirow[b]{2}{*}{ Raça/Cor da pele } & \multicolumn{5}{|c|}{ Renda familiar per capita em frações do salário mínimo } & \multirow[b]{2}{*}{ Total } \\
\hline & 0 a I/2 & $\begin{array}{c}\text { mais de } \\
\mathrm{I} / 2 \text { a } \mathrm{I}\end{array}$ & $\begin{array}{c}\text { mais de } \\
\text { I a } 2\end{array}$ & $\begin{array}{c}\text { mais de } \\
2 \text { a } 5\end{array}$ & mais de 5 & \\
\hline Brancos & $12,4 \%$ & $15,3 \%$ & $26,5 \%$ & $52,9 \%$ & $81,0 \%$ & $39,5 \%$ \\
\hline Não-brancos & $4,7 \%$ & $8,2 \%$ & $18,2 \%$ & $41,2 \%$ & $70,4 \%$ & $18,8 \%$ \\
\hline Total & $7,9 \%$ & $11,9 \%$ & $23,4 \%$ & $50,4 \%$ & $79,5 \%$ & $32,2 \%$ \\
\hline
\end{tabular}

Fonte: PNAD, 2003. 
FIGURA 4

PORCENTAGEM DOS JOVENS DE I 8 A 24 ANOS COM ACESSO AO ENSINO SUPERIOR ENTRE OS QUE CONCLUÍRAM O ENSINO MÉDIO, SEGUNDO A RENDA DOMICILIAR PER CAPITA E RAÇA/COR

BRASIL, 2003

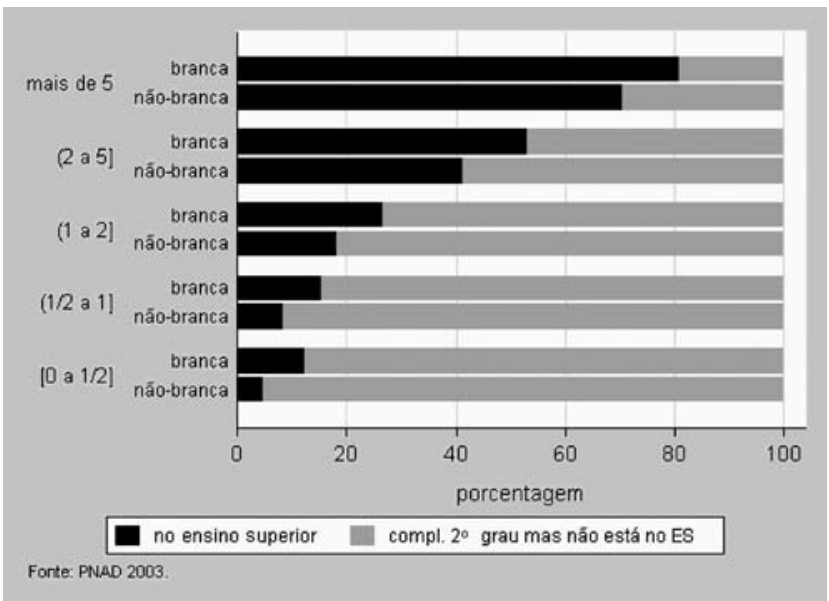

TABELA 5

DISTRIBUIÇÃO DA POPULAÇÃO DE I 8 A 24 ANOS QUE CURSA OU CURSOU ENSINO SUPERIOR SEGUNDO A REDE DE ENSINO E A RENDA FAMILIAR PER CAPITA

BRASIL, 2003

\begin{tabular}{lcccccc}
\hline & \multicolumn{3}{c}{$\begin{array}{c}\text { Distribuição de acordo com } \\
\text { faixa de renda per capita }\end{array}$} & \multicolumn{3}{c}{$\begin{array}{c}\text { Distribuição de acordo com } \\
\text { tipo da rede de ensino }\end{array}$} \\
\cline { 2 - 7 } Faixas de Renda & Privada & Pública & Total & Privada & Pública & Total \\
\hline < 1/2 SM & $2 \%$ & $5 \%$ & $3 \%$ & $53 \%$ & $47 \%$ & 64.952 \\
I /2 a I SM & $6 \%$ & $11 \%$ & $8 \%$ & $58 \%$ & $42 \%$ & 181.644 \\
I a 2 SM & $22 \%$ & $28 \%$ & $23 \%$ & $67 \%$ & $33 \%$ & 561.744 \\
2 a 5 SM & $45 \%$ & $37 \%$ & $43 \%$ & $76 \%$ & $24 \%$ & 1.038 .809 \\
mais de 5 SM & $25 \%$ & $19 \%$ & $23 \%$ & $78 \%$ & $22 \%$ & 560.905 \\
\hline Total & 1.742.057 & 665.997 & 2.408 .054 & $72 \%$ & $28 \%$ & 2.408 .054 \\
\hline
\end{tabular}

Fonte: PNAD/BGE. 
dos na rede privada e $34 \%$ dos matriculados na rede pública, mostrando que na rede pública os níveis de iniqüidade são menores.

Quanto à distribuição dos matriculados por tipo de rede para cada nível salarial, observa-se que em todas as faixas de renda per capita do domicílio há mais alunos de ensino superior em estabelecimentos privados do que em estabelecimentos da rede pública, mas essa porcentagem maior na rede privada aumenta substancialmente quando a renda aumenta, ultrapassando de $53 \%$ dos alunos na faixa de menos de meio salário mínimo per capita a 78\% na faixa de mais de cinco salários mínimos per capita.

Essa constatação contraria o senso comum, amplamente divulgado pela imprensa, de que "os ricos estudam nas universidades públicas e gratuitas, enquanto os pobres têm acesso quase que exclusivamente ao ensino superior privado".

Os dados apresentados na tabela 6 mostram a distribuição das matrículas do ensino superior público e privado segundo a condição de raça/cor da população. Quando se observa a distribuição das matrículas públicas e privadas entre os jovens brancos e não-brancos, verifica-se que o contingente de pardos e negros matriculados no ensino superior representa $20,54 \%$ do total dos jovens matriculados, sendo que no ensino público são $27 \%$ e no ensino privado $18 \%$.

O gráfico da figura 5 apresenta as distribuições cumulativas de matrículas no ensino superior de jovens de 18 a 24 anos em estabelecimentos públicos e privados. No mesmo gráfico, para fins de comparação, apresenta-se a curva de Lorenz para a renda, sendo esta a distribuição cumulativa da renda per capita nesse mesmo grupo de idade.

TABELA 6

PERCENTUAL DA POPULAÇÃO DE I 8 A 24 ANOS CURSANDO ENSINO SUPERIOR SEGUNDO A REDE DE ENSINO E A RAÇA/COR BRASIL , 2003

\begin{tabular}{lccccccccc}
\hline Rede de ensino & & Pública & \multicolumn{3}{c}{ Privada } & & \multicolumn{2}{c}{ Total } \\
\hline \multicolumn{1}{c}{ Raça/cor } & Brancos & $\begin{array}{c}\text { Não- } \\
\text { brancos }\end{array}$ & Total & Brancos & $\begin{array}{c}\text { Não- } \\
\text { brancos }\end{array}$ & Total & Brancos & $\begin{array}{c}\text { Não- } \\
\text { brancos }\end{array}$ & Total \\
\hline Total & 486.988 & 179.009 & 665.997 & 1.426 .547 & 315.510 & 1.742 .057 & 1.913 .535 & 494.519 & 2.408 .054 \\
\hline Porcentagem & $73 \%$ & $27 \%$ & $100 \%$ & $82 \%$ & $18 \%$ & $100 \%$ & $79 \%$ & $21 \%$ & $100 \%$ \\
\hline Fonte: PNAD 2003. & & & & & & & &
\end{tabular}


FIGURA 5

DISTRIBUIÇÃO DA RENDA PER CAPITA E DAS MATRIICULAS DO ENSINO SUPERIOR TOTAL E PÚBLICO BRASIL, 2003

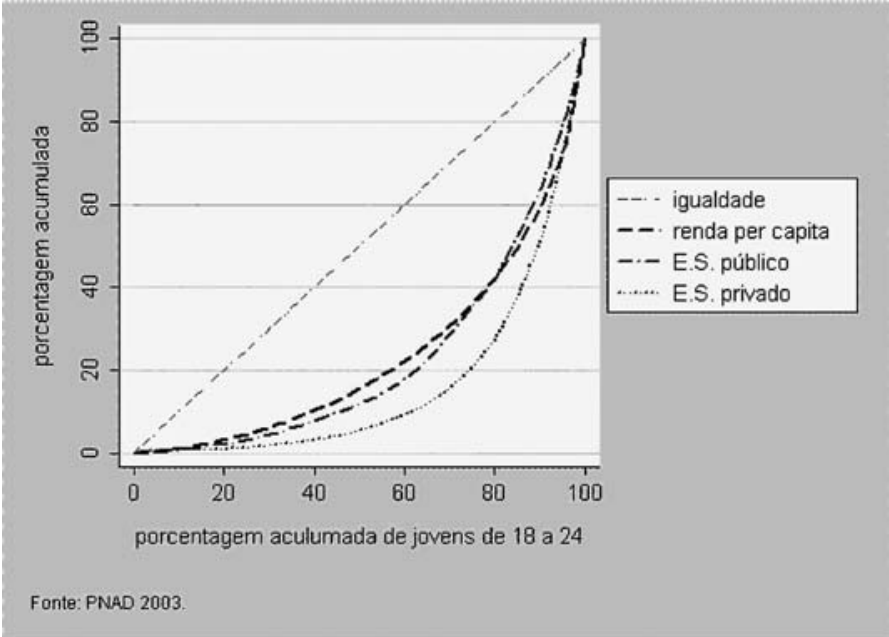

\section{CONCLUSÕES E DISCUSSÃO}

Apenas 13\% dos jovens de 18 a 24 anos têm acesso ao ensino superior. Neste sentido, importa identificar o que ocorre com o contingente que não atinge esse nível de ensino, considerando as faixas etárias anteriores, as transições escolares, bem como as clivagens derivadas da renda e da cor.

O principal determinante do acesso à educação e da progressão dentro de padrões de adequação, para uma relação entre idade curso e série, é a renda familiar, embora a variável raça/cor também apresente influência em todas as faixas de renda. Há um importante efeito cumulativo devido ao atraso e à evasão escolar nas camadas de menor renda, que leva a enorme diferença nos percentuais de acesso aos níveis mais altos de ensino.

A desigualdade por renda e cor aumenta com a progressão escolar. No grupo de sete a dez anos de idade, as diferenças entre os diferentes níveis de renda e entre brancos e não-brancos são bastante pequenas, devido à quase universalização do ensino fundamental no país para essas idades. No grupo de 
Acesso à educação por faixas etárias...

dez a 14 anos já surge o padrão de desigualdades que irá aumentar com a idade e a progressão na escola. As diferenças aumentam entre os extremos das faixas de renda, as desigualdades entre brancos e não-brancos são menores nos grupos de renda mais altos e se acentuam para os níveis de renda mais baixos.

Neste último grupo de idade, as porcentagens de crianças que estão em uma etapa "adequada" na progressão escolar, segundo os critérios usados neste trabalho, são de aproximadamente $97 \%$ para brancos e $93 \%$ para não-brancos, na faixa de mais de cinco salários mínimos de renda per capita, mas são apenas de aproximadamente $72 \%$ para os brancos e de $57 \%$ para os não-brancos na faixa de renda per capita até meio salário mínimo. A razão entre as porcentagens para brancos e não-brancos é de apenas 1,05 para a faixa mais alta de renda, mas sobe a I,26 na faixa de renda mais baixa. Já entre os extremos das faixas de renda, essas razões são de 1,35 para brancos e 1,63 para não-brancos. Em outras palavras, existe um efeito mais do que cumulativo, com um sinergismo entre renda e cor da pele extremamente perverso.

Esse sinergismo e as desigualdades, como foi dito, acentuam-se com a idade e a progressão escolar. No grupo de adolescentes de 15 a 17 anos de idade as porcentagens de adequação são de aproximadamente $91 \%$ para brancos e $90 \%$ para não-brancos no grupo de maiores rendas, caindo respectivamente para cerca de $40 \%$ e $27 \%$ na faixa de rendas mais baixas. As razões entre essas porcentagens são de apenas 1,0 I entre brancos e não-brancos na faixa de rendas superiores, aumentando para I,48 na faixa de rendas mais baixas. Deve-se notar que nesta faixa, abaixo de meio salário mínimo, a razão entre brancos e não-brancos era de 1,26 no grupo etário anterior. Entre os brancos a razão entre os extremos de faixas de renda é agora de aproximadamente 2,28 e entre não-brancos, de mais de 3,32.

Para o último grupo de idades considerado, no qual se tomou como "adequado" ter tido acesso ao ensino superior, a situação é obscena. Para brancos na faixa de mais de cinco salários mínimos per capita de renda domiciliar, a porcentagem de jovens que alcançam este nível de ensino é de aproximadamente $73 \%$ e entre os não-brancos, de 55\%. Já na faixa de até meio salário, as porcentagens são de 2,2\% para brancos e 0,5\% para não-brancos. As razões são portanto de aproximadamente 1,33 entre brancos e não-brancos na faixa de maiores rendas, aumentando para 4,4 na faixa de rendas até meio salário mínimo. Para os brancos a razão entre os extremos de renda é de 33,2 
e para os não-brancos, de I | 0 . Este comportamento é apresentado de forma resumida nas figuras I e 2 .

Ao se analisar especificamente o grupo de 18 a 24 anos de idade, essas desigualdades crescentes e esse sinergismo entre renda e cor da pele se manifestam num panorama de profundas disparidades na situação em que se encontram estes indivíduos em termos do maior nível de escolaridade que atingiram (Tab. 1, 2 e 3; Fig. 3).

No grupo de menores rendas, $60 \%$ desses jovens não chegaram a concluir o ensino fundamental e outros $26 \%$ não concluíram o ensino médio, enquanto para a faixa de rendas superiores a cinco salários mínimos apenas 3\% não concluíram o ensino fundamental e outros $6 \%$ não concluíram o ensino médio. Apenas $1 \%$ destes jovens chegaram ao ensino superior no grupo de rendas per capita de até meio salário mínimo, enquanto na faixa de mais de cinco salários mínimos esta porcentagem chega a 71\%. Para o grupo completo (todas as faixas de renda), $34 \%$ não concluíram o ensino fundamental e outros $26 \%$ não concluíram o ensino médio. Apenas cerca de 13\% tiveram acesso ao ensino superior, como já mencionado. Essas porcentagens, muito mais próximas dos valores das faixas de renda mais baixas, refletem o fato de que, dos pouco mais de 22,4 milhões de jovens nestas idades no Brasil, mais da metade ( 12,7 milhões aproximadamente) estão em domicílios com rendas per capita de até um salário mínimo.

Ao compararmos brancos e não-brancos, as desigualdades são bem menores, reflexo do fato que, em todas as idades anteriores, as diferenças por renda foram sempre bastante superiores às disparidades por cor da pele. São $44 \%$ os não-brancos que não completaram o ensino fundamental, enquanto para brancos essa porcentagem é de $23 \%$. As porcentagens dos que completaram esta etapa, mas não concluíram o ensino médio, são muito parecidas, de 27\% e 25\%, respectivamente. A maior desigualdade é no ensino superior, quando as porcentagens são de $21 \%$ para brancos e de apenas $5 \%$ para negros.

É necessário, no entanto, estudar com cuidado estas cifras, uma vez que entre nível baixo de renda e cor da pele não-branca existiu um sinergismo perverso em todas as etapas da evolução no sistema escolar.

Ao examinar o panorama da situação de maior nível de escolaridade combinando os efeitos de renda e cor da pele notamos que as desigualdades mais importantes são de fato relacionadas com a renda e não com a cor da pele. 
Acesso à educação por faixas etárias...

Entre os brancos a razão entre as porcentagens de jovens de 18 a 24 anos, que concluíram o ensino médio nos extremos das faixas de renda, é de aproximadamente 4,8 (90,4\% / 18,7\%) enquanto entre os não-brancos esta razão é de pouco menos de 6,6. Na faixa de rendas de mais de cinco salários mínimos a razão entre brancos e não-brancos é de 1,15 , enquanto na faixa de até meio salário mínimo de renda per capita ela alcança o valor de 1,56.

As desigualdades menores dentro de cada faixa de renda, ainda que crescentes para rendas menores, entre brancos e não-brancos, não se refletem no geral nesse grupo populacional, devido às grandes diferenças na distribuição de brancos e não-brancos nas diferentes faixas de renda. Aproximadamente metade desses jovens são brancos. Dos pouco mais de I I milhões de jovens brancos, cerca de 4,8 milhões estão em domicílios com rendas até um salário mínimo per capita, enquanto entre os também aproximadamente I I milhões de não-brancos a cifra alcança quase 8 milhões. Existem pouco mais de 830 mil jovens na faixa de mais de cinco salários mínimos per capita, representando cerca de 7,3\% do total, mas apenas I 50 mil jovens não-brancos nestas condições, o que representa menos de 1,5\%.

A transição para o ensino superior neste grupo de idades apresenta de novo características semelhantes ao que já se observou nas idades mais baixas, ou seja, as grandes desigualdades ocorrem entre os extremos de rendas, com desigualdades menores entre brancos e não-brancos.

Do grupo de jovens que tem o requisito formal básico para poder aspirar ao acesso ao ensino superior, cerca de $32 \%$ o fazem, sendo que essa porcentagem é de quase $40 \%$ entre os brancos e um pouco inferior a 19\% entre os não-brancos (Tab. 4). Ao examinar o comportamento desse acesso por faixas de renda vemos que é de quase $80 \%$ para rendas de mais de cinco salários mínimos per capita caindo a menos de $8 \%$ na faixa de até meio salário mínimo per capita. A desigualdade grande entre brancos e não-brancos na população geral desse grupo não ocorre dentro de cada faixa de rendas per capita, sendo sim, de novo, reflexo da diferença da distribuição dos dois grupos de acordo com a renda. Na faixa de mais de cinco salários mínimos as porcentagens de acesso em relação aos graduados no ensino médio são de $81 \%$ para os brancos e pouco mais de 70\% para os não-brancos, com uma razão de apenas I, I 5. Na faixa de até meio salário essas porcentagens caíram 12,4\% para brancos e 4,7\% para não-brancos, com uma razão entre elas de aproxi- 
madamente 2,6. Mas se examinarmos o que ocorre entre os extremos de renda temos uma razão de 6,5 para brancos e de quase 15 para não-brancos. Ou seja, exatamente como antes, as grandes desigualdades se observam entre os extremos da renda, sendo maior entre não-brancos do que brancos o sinergismo já comentado.

É importante ressaltar que essa transição entre graduação no ensino médio e o ensino superior apresenta, no Brasil, características globais que são comparáveis às de países da Europa Ocidental e dos Estados Unidos e Canadá. No país, neste grupo de jovens de 18 a 24 anos encontramos um contingente de pessoas que tiveram acesso ao ensino superior que é igual a cerca de $40 \%$ do contingente que completou o ensino médio. Nos países da Europa Ocidental as taxas de acesso ao ensino superior entre jovens que completaram o ensino médio variam tipicamente entre $30 \%$ e $50 \%$ na maioria dos casos, enquanto nos Estados Unidos alcançaram quase 64\% no ano de 2003 (Organização para a Cooperação e Desenvolvimento Econômico - OCDE, 2006; United States, 2005). No caso dos Estados Unidos, em que se estudou o acesso por níveis de renda e grupo étnico, observam-se desigualdades no acesso bem menores do que no Brasil, tanto em relação a grupo étnico quanto a níveis de renda. Entre os brancos a porcentagem de jovens que se graduaram na high schoole se matricularam no ensino superior foi de pouco mais de 66\%, caindo para cerca de 58\% entre afro-americanos e 59\% entre hispânicos. Quanto à situação econômica, nos 20\% de menores rendas per capita, o acesso foi de $53 \%$, nos $20 \%$ de maiores rendas, cerca de $80 \%$ e nos $60 \%$ intermediários de aproximadamente 58\%.

Finalmente se pode observar que pessoas das faixas de menor renda têm pouco acesso ao ensino superior em geral, mas quando esse acesso ocorre, ele se dá mais freqüentemente no ensino superior público do que no privado. Essa análise para os dois grupos de estabelecimentos torna-se muito relevante no país diante da recente expansão das matrículas em estabelecimentos de terceiro grau, principalmente no setor privado (Andrade, 2004).

A população de jovens de 18 a 24 anos de idade com renda familiar per capita até um salário mínimo per capita, na verdade, tem pouquíssima participação no ensino superior, quer no setor privado, quer no público, embora apresente uma participação proporcionalmente maior no setor público. Dito de outra forma, pessoas das faixas de menor renda têm pouco acesso ao ensi- 
no superior em geral, mas quando têm acesso, este se dá mais freqüentemente no ensino público do que no privado. Sampaio, Limongi e Torres (2000) apontam para conclusões semelhantes quando observam os alunos participantes do Exame Nacional de Cursos.

Pode-se fazer, portanto, outra constatação, de grande importância, mais uma vez contrária a idéia corrente de que "pessoas das faixas de menor renda e não brancas têm mais acesso ao ensino superior privado enquanto pessoas das faixas de renda mais alta e brancas estudam no setor público e gratuito". Em que pese o fato de que não-brancos sejam a minoria dos alunos no ensino superior, eles representam um contingente proporcionalmente maior no setor público do que no setor privado, quando comparados aos brancos.

A análise das curvas de distribuição cumulativas de matriculados no ensino superior mostra que a curva que corresponde ao ensino público está muito próxima da curva cumulativa de renda, ainda que passe abaixo para rendas mais baixas e acima da renda, para rendas mais altas. A curva para o ensino privado é bastante "regressiva" em relação à renda, estando abaixo da curva de renda para toda a gama de rendas per capita.

Esta constatação confirma o que foi comentado: o ensino superior público é menos injusto no país em termos de renda do que o ensino privado. Ainda assim a situação não é satisfatória. É fundamental que a curva cumulativa para o ensino público se torne "progressiva", ou seja, que esteja acima da curva de renda, para que o acesso ao ensino superior público se transforme em um dos instrumentos de mobilidade social ascendente no país. A maior ou menor regressividade da curva de matrículas no ensino superior público parece estar fortemente associada à forma da curva de graduação no ensino médio (Dachs, Andrade, 2006).

Estes resultados mostram que a adoção de políticas de cotas raciais nas universidades públicas do país pode ter um efeito pernicioso de aumentar ainda mais as desigualdades entre não-brancos ao ensino superior no Brasil. Será necessário associar políticas afirmativas para esse grupo às condições econômicas do grupo familiar, uma vez que se observa que o maior gradiente de acesso é decorrente da renda e não das diferenças raciais e étnicas. Programas indiscriminados de cotas raciais irão aumentar o contingente de não-brancos nas escolas de nível superior provenientes das faixas de rendas mais altas. Nos níveis mais baixos simplesmente não existem jovens graduados no ensino médio 
em número suficiente e com as qualificações acadêmicas necessárias para cruzar essa transição em que se observa o maior gargalo no sistema educacional brasileiro. Mais do que cotas nas universidades, as políticas públicas deveriam preocupar-se em diminuir as perdas sucessivas ao longo do sistema educacional, especialmente dos alunos nas faixas de menores rendas, nas quais se concentram majoritariamente as crianças e jovens brasileiros não-brancos.

\section{REFERÊNCIAS BIBLIOGRÁFICAS}

ANDRADE, C. Y. Ensino superior: expansão e desafios. Com Ciência: Revista Eletrônica de Jornalismo Cientifico, n.54, maio 2004.

BARROS, R. P. de et al. Determinantes do desempenho educacional no Brasil. Rio de Janeiro: Ipea, 200I. (Texto para Discussão, 834)

BELTRÃO, K. I. Acesso à educação: diferenciais entre os sexos. Rio de Janeiro, 2002. (Texto para Discussão, 879)

Alfabetização por sexo e raça no Brasil: um modelo linear generalizado para explicar a evolução no período 1940-2000. Rio de Janeiro: Ipea, 2003. (Texto para Discussão, 1003)

BELTRÃO, K. I.; TEIXEIRA, M. de P. O Vermelho e o negro: raça e gênero na universidade brasileira - uma análise da seletividade das carreiras a partir dos censos demográficos de 1960 a 2000. Rio de Janeiro: Ipea, 2004. (Texto para Discussão, I.052)

BRANDÃO, A. A.; MARINS, M. T. A. de. Nos Labirintos da política de cotas para negros no ensino superior. Physis, v. 15, p. 165-170, 2005.

CAMARANO, A. A. et al. Caminhos para a vida adulta: as múltiplas trajetórias dos jovens brasileiros. Rio de Janeiro: Ipea, 2004. (Texto para Discussão, I.038)

CORSEUIL, Ca. H. et al. Decisões críticas em idades críticas. a escolha dos jovens entre estudo e trabalho no Brasil e em outros países da América Latina. Rio de Janeiro: Ipea, 200 I. (Texto para Discussão, 797)

CRUZ, C. H. B. Educação e desenvolvimento: anotações sobre mitos e verdades sobre educação no Brasil. In: ENCONTRO ANUAL DA ACADEMIA BRASILEIRA DE CIÊNCIAS. Rio de Janeiro, 5 jun. 2006. Disponível em: http://www.ifi.unicamp.br/ brito/artigos/ educacao-desenvolvimento-abc-usadas-I.pdf. Acesso em: 8 nov. 2006. 
Acesso à educação por faixas etárias...

DACHS, J. N. W.; ANDRADE, C. Y. Distribuição de renda dos jovens de 18 a 24 anos ensino superior, de acordo com o tipo de estabelecimento (público ou privado), as cotas alternativas, 2006. submetido a publicação.

FERNANDES, D. C. Estratificação educacional, origem socioeconômica e raça no Brasil: as barreiras da cor. (Concurso de Monografia, Prêmio Ipea 40 anos, IPEA-CAIXA, 2004)

FERRARO, A. R.; MACHADO, N. C. F. Da universalização do acesso à escola no Brasil. Educação \& Sociedade, v.23, n.79, p.213-2|4, 2002.

GUIMARÃES, A. S. A. Acesso de negros às universidades públicas. Cadernos de Pesquisas, n. I I8, p.247-268, mar.2003.

HASENBALG, C. e S.; SILVA, N. do. V. Família, cor e acesso à escola no Brasil. In: HASENBALG, C. S.; V. SILVA, N. do V.; LIMA, M. Cor e estratificação social no Brasil. Rio de Janeiro: Contra Capa, 1999. p. 126-147.

HENRIQUES, R. Desigualdade racial no Brasil: evolução das condições de vida na década de 90. Rio de Janeiro: Ipea, 200।. (Texto para Discussão, 807)

JACCOUD, L.; BEGHIN, N. Desigualdades raciais no Brasit: um balanço da intervenção governamental. Brasília: Ipea, 2002.

MAGGIE, Y.; FRY, P. A Reserva de vagas para negros nas universidade brasileiras. Estudos Avançados, v. 18, n.50, p.67-80, 2004.

MAIO, M. C.; SANTOS, R. V. Política de cotas raciais, os "olhos da sociedade" e os usos da antropologia: o caso do vestibular da Universidade de Brasília (UnB). Horizontes Antropológicos, v. I I, n.23, p. I8I-2|4, 2005.

MOELECKE, S. Ação afirmativa: história e debates no Brasil. Cadernos de Pesquisa, São Paulo, n. I17, p.197-217, nov. 2002.

ORGANIZATION FOR ECONOMIC COOPERATION AND DEVELOPMENT - OECD. Education at a glance 2006. Paris, 2006.

OLIVEIRA, F. Ser negro no Brasil: alcances e limites. Estudos Avançados, v. I 8, n.50, p.57-60, 2005.

OSORIO, R. G. A Mobilidade social dos negros brasileiros. Rio de Janeiro, Ipea, 2004. (Texto para Discussão, 1.033)

PEDROSA, R. H. L. et al. Educational and socioeconomic background of undergraduates and academic performance: consequences of affirmative action programs at a brazilian research university. (Apresentado na General Conference of the Institutional Management in Higher 
Education, OECD, Paris, 2006; submetido a publicação em Higher Education Management and Policy)

ROCHA, S. Pobreza no Brasil: do que se trata. Rio de Janeiro: Editora FGV, 2003.

SAMPAIO, H.; LIMONGI, F.; TORRES, H. Eqüidade e heterogeneidade no ensino superior brasileiro. Brasília: Instituto Nacional de Estudos e Pesquisas Educacionais, 2000.

SCHWARTZMAN, S. Fora de foco: diversidade e identidades étnicas no Brasil. Novos Estudos Cebrap, n.55, p.83-96, 1999.

SOARES, J. F.; ALVES, T. G. Desigualdades raciais no sistema brasileiro de educação básica. Educação e Pesquisa, São Paulo, v.29, n. I, 2003. Disponível em: http://www.scielo.br/pdf/ ep/v29n l/a l Iv29nl.pdf . Acesso em: out. 2006.

SOARES, S.; CARVALHO, L.; KIPNIS, B. Os Jovens de 18 a 25 anos. retrato de uma dívida da política educacional. Rio de Janeiro: Ipea, 2003. (Texto para Discussão, 954)

SOARES, S. et al. (orgs) Os Mecanismos de discriminação racial nas escolas brasileiras. Rio de Janeiro: Ipea, 2005.

TRAVASSOS, C.; WILLIAMS, D. R. The Concept and measurement of race and their relationship to public health: a review focused on Brazil and the United States. Cadernos de Saúde Pública, v.20, n.3, p.660-678, 2004.

UNITED STATES. Department Of Education. National Center for Education Statistics. The Condition of education 2005. Whashington: Government Printing Office, 2005-094.

ZUCHI, J. Desigualdade de renda no Brasil em 200/: a influência da cor e da educação. (Concurso de Monografias, Prêmio IPEA 40 anos, IPEA-CAIXA, 2004. Tema: A Superação das Desigualdades no Brasil)

Recebido em: outubro 2006

Aprovado para publicação em: dezembro 2006 